\title{
ON POLYNOMIALLY BOUNDED OPERATORS WITH RICH SPECTRUM
}

\author{
RADU GADIDOV
}

(Communicated by Palle E. T. Jorgensen)

\begin{abstract}
D. Westood (J. Funct. Anal. 66 (1986), 96-104) proved that $C_{00}$ contractions with dominating spectrum are in $A_{\aleph_{0}}$. We generalize this result to polynomially bounded operators.
\end{abstract}

\section{INTRODUCTION}

Let $\mathscr{H}$ be a complex, separable, infinite dimensional Hilbert space, and let $\mathscr{B}(\mathscr{H})$ be the algebra of all bounded, linear operators on $\mathscr{H}$. Recall that an operator $T \in \mathscr{B}(\mathscr{H})$ is called polynomially bounded (notation $T \in(P B)(\mathscr{H})$ ) if there exists a constant $K \geq 1$ such that for every polynomial $p$,

$$
\|p(T)\| \leq K \sup \{|p(z)|:|z|=1\} .
$$

Of course, all contraction operators in $\mathscr{B}(\mathscr{H})$ are polynomially bounded, and in the past fifteen years the theory of dual algebras generated by a single contraction operator has been used very succesfully to obtain information about the structure of such operators (see for example [1], [2], [5], [6]). More recently (cf. [11], [12], [13], [15], etc.), researchers have begun to use the theory of dual algebras generated by an arbitrary polynomially bounded operator to extract structural information about such operators. As was pointed out in [11], however, many parts of the theory for contraction operators do not readily generalize to the case of polynomially bounded operators. The purpose of this note is to make a modest contribution to this theory, by proving a generalization (Theorem 2 below) of the main result in [16] and one of the results in [11]. Before stating Theorem 2, we recall some notation and definitions from this theory.

If $T$ is in $\mathscr{B}(\mathscr{H})$ and $\mathscr{K}$ is a (closed) subspace of $\mathscr{H}$, then $T_{\mathscr{K}}$ denotes the compression of $T$ to $\mathscr{M}$, i.e., $T_{\mathscr{M}}=P_{\mathscr{M}} T_{\mathscr{M}}$, where $P_{\mathscr{K}}$ denotes the orthogonal projection from $\mathscr{H}$ onto $\mathscr{M}$. Also the spectrum of $T$, the point spectrum of $T$ and the essential spectrum of $T$ will be denoted by $\sigma(T)$, $\sigma_{p}(T)$ and $\sigma_{e}(T)$, respectively. Moreover, $C_{00}(\mathscr{H})$ is the set of all operators $\mathrm{T}$ in $\mathscr{B}(\mathscr{H})$ such that the sequences $\left\{T^{n}\right\}_{n=1}^{\infty},\left\{T^{* n}\right\}_{n=1}^{\infty}$ converge to 0 in the strong operator topology on $\mathscr{B}(\mathscr{H})$.

Received by the editors August 23, 1993 and, in revised form, October 27, 1993.

1991 Mathematics Subject Classification. Primary 47A15; Secondary 47A60.

(C)1995 American Mathematical Society 
It is well known (cf. [9]) that $\mathscr{B}(\mathscr{H})$ is the dual space of the Banach space $\mathscr{C}_{1}(\mathscr{H})$ of trace-class operators on $\mathscr{H}$ equipped with the trace-norm \|\|$_{1}$, and the duality is implemented by the bilinear form $\langle T, L\rangle=\operatorname{trace}(\mathrm{TL}), T \in$ $\mathscr{B}(\mathscr{H}), L \in \mathscr{C}_{1}(\mathscr{H})$. If $T$ is an operator in $\mathscr{B}(\mathscr{H}), \mathscr{A}_{T}$ will denote the dual algebra generated by $T$ (i.e., the smallest weak ${ }^{*}$-closed algebra containing $T$ and the identity operator on $\mathscr{H}), \mathscr{Q}_{T}\left(=\mathscr{C}_{1} /{ }^{\perp} \mathscr{A}_{T}\right)$ the natural predual of $\mathscr{A}_{T}$. For any $L \in \mathscr{C}_{1}(\mathscr{H})$ the corresponding element in $\mathscr{Q}_{T}$ will be denoted by $[L]_{T}$. In particular, for any vectors $x$ and $y$ in $\mathscr{H},[x \otimes y]_{T}$ is the image in $\mathscr{Q}_{T}$ of $x \otimes y$, where $x \otimes y$ denotes the usual rank one operator in $\mathscr{B}(\mathscr{H})$.

As usual $\mathbf{D}$ denotes the open unit disc in $\mathbf{C}$, and $\mathbf{T}=\partial \mathbf{D}$. If $\mathrm{E}$ is a measurable subset of $\mathbf{T}$ (with respect to normalized Lebesgue measure $\mathbf{m}$ on $\mathbf{T}$ ), a set $\Lambda \subset \mathbf{D}$ is said to be dominating for $E$ if almost every point of $E$ is a nontangential limit of a sequence of points from $\Lambda$, and the set of all nontangential limits of $\Lambda$ on $\mathbf{T}$ will be denoted by $N T L(\Lambda)$. The spaces $\mathbf{L}^{1}\left(:=\mathbf{L}^{1}(\mathbf{T})\right), \mathbf{H}^{1}\left(:=\mathbf{H}^{1}(\mathbf{T})\right)$ and $\mathbf{H}^{\infty}\left(:=\mathbf{H}^{\infty}(\mathbf{T})\right)$ are the usual Lebesgue and Hardy function spaces on $\mathbf{T}$, relative to the measure $\mathbf{m}$. It is easy to see that if $T \in(P B)(\mathscr{H})$, there exists a smallest number $\mathrm{M}$ such that (1) is valid for every polynomial $p$, and we denote the set of all $T \in(P B)(\mathscr{H})$ for which $\mathrm{M}$ is the smallest such number by $(P B)^{M}(\mathscr{H})$ (cf. [11]). If $T \in(P B)^{M}(\mathscr{H})$, it is easy to see that for any pair of vectors $x$ and $y \in \mathscr{H}$ there exists a measure $\mu_{x, y}$ on $\mathrm{T}$ such that for every polynomial $p$,

$$
\langle p(T) x, y\rangle=\int_{\mathbf{T}} p d \mu_{x, y},
$$

and the operator $\mathrm{T}$ is called absolutely continuous (notation $T \in(A C P B)^{M}(\mathscr{H})$ ) if for every pair $x, y$ in $\mathscr{H}$ there exists an absolutely continuous measure $\mu_{x, y}$ satisfying (2) (with respect to $\mathbf{m}$ ).

For absolutely continuous polynomially bounded operators it is well known (cf. [11]) that there exists a unique unital, norm continuous algebra homomorphism

$$
\Phi_{T}: \mathbf{H}^{\infty} \rightarrow \mathscr{A}_{T}
$$

onto a weak ${ }^{*}$ dense subalgebra of $\mathscr{A}_{T}$ such that $\Phi_{T}$ extends the Riesz-Dunford functional calculus, $\Phi_{T}$ is continuous if both $\mathbf{H}^{\infty}$ and $\mathscr{A}_{T}$ are given their weak *-topologies, and $\Phi_{T}$ is the adjoint of a bounded, linear, one to one map

$$
\phi_{T}: \mathscr{Q}_{T} \rightarrow \mathbf{L}^{1} / \mathbf{H}_{0}^{1} \text {. }
$$

Let us also recall (cf. [11]) that the class $\mathbb{A}^{M}(\mathscr{H})$ is the set of all $T \in$ $(A C P B)^{M}(\mathscr{H})$ for which $\Phi_{T}$ is bounded below. In this case $\Phi_{T}$ is also a weak $^{*}$ homeomorphism between $\mathbf{H}^{\infty}$ onto $\mathscr{A}_{T}$, when $\mathbf{H}^{\infty}$ and $\mathscr{A}_{T}$ are given their weak *-topologies, and $\phi_{T}$ is onto.

For any $f$ in $\mathbf{L}^{1},[f]_{\mathbf{L} / \mathbf{H}_{0}^{1}}$ denotes the image of $f$ in $\mathbf{L} / \mathbf{H}_{0}^{1}$ under the canonical projection from $\mathbf{L}^{1}$ onto $\mathbf{L}^{1 / \mathbf{H}_{0}^{1}}$. If $\lambda \in \mathbf{D}$ and $\mathbf{P}_{\lambda}$ is the associated Poisson kernel on $\mathbf{T}$ (i.e., $\mathbf{P}_{\lambda}(t):=\frac{\left(1-|\lambda|^{2}\right)}{\mid 1-\bar{\lambda} e^{\left.i\right|^{2}}}$ ), we write

$$
\left[C_{\lambda}\right]_{T}=\phi_{T}^{-1}\left(\left[\mathbf{P}_{\lambda}\right]_{\mathbf{L} / \mathbf{H}_{0}^{1}}\right),
$$

and it is easy to check that for any function $h$ in $\mathbf{H}^{\infty}$,

$$
\left\langle\Phi_{T}(h),\left[C_{\lambda}\right]_{T}\right\rangle=h(\lambda) .
$$


If $T \in \mathbb{A}^{M}(\mathscr{H})$, then, as is customary, $\mathscr{E}_{0}\left(\mathscr{A}_{T}\right)$ denotes the set of all $[L]_{T}$ in $\mathscr{Q}_{T}$ for which there exist sequences $\left\{x_{n}\right\}_{n=1}^{\infty},\left\{y_{n}\right\}_{n=1}^{\infty}$ in the unit ball of $\mathscr{H}$ such that

(i) $\lim _{n \rightarrow \infty}\left\|[L]_{T}-\left[x_{n} \otimes y_{n}\right]_{T}\right\|=0$, and

(ii) $\lim _{n \rightarrow \infty}\left(\left\|\left[x_{n} \otimes w\right]_{T}\right\|+\left\|\left[w \otimes y_{n}\right]_{T}\right\|\right)=0$ for any $w \in \mathscr{H}$,

and $\mathbb{A}^{M}(\mathscr{H})$ has property $\mathscr{X}_{0, \theta}(\theta \in(0,1])$ if $\mathscr{E}_{0}\left(\mathscr{A}_{T}\right)$ (which is (cf. [4]) absolutely convex and norm closed) contains the closed ball in $\mathscr{Q}_{T}$ centered at 0 with radius $\theta$.

The following result comes from [11], and will be needed in the sequel.

Lemma 1. Let $T \in \mathbb{A}^{M}(\mathscr{H}) \cap C_{00}(\mathscr{H})$.

(i) If $\left\{x_{n}\right\}_{n=1}^{\infty}$ is a sequence of vectors converging weakly to 0 , then for any vector $z \in \mathscr{H}$,

$$
\lim _{n \rightarrow \infty}\left(\left\|\left[x_{n} \otimes z\right]_{T}\right\|+\left\|\left[z \otimes x_{n}\right]_{T}\right\|\right)=0 .
$$

(ii) If $\lambda \in \sigma_{e}(T) \cap \mathbf{D}$, then $\left[C_{\lambda}\right]_{T} \in \mathscr{E}_{0}\left(\mathscr{A}_{T}\right)$.

Finally, we write, as is customary, $\mathbb{A}_{\aleph_{0}}^{M}(\mathscr{H})$ for the set of those operators $T$ in $\mathbb{A}^{M}(\mathscr{H})$ such that for any doubly indexed sequence $\left\{\left[L_{i j}\right]_{T}\right\}_{i \geq 1 ; j \geq 1}$ of elements of $\mathscr{Q}_{T}$, there exist sequences $\left\{x_{i}\right\}_{i=1}^{\infty}$ and $\left\{y_{j}\right\}_{j=1}^{\infty}$ of vectors in $\mathscr{H}$ such that

$$
\left[L_{i j}\right]_{T}=\left[x_{i} \otimes y_{j}\right]_{T}, \quad 1 \leq i, \quad 1 \leq j .
$$

Now we may state the main result of this note.

Theorem 2. Let $T \in(P B)^{M}(\mathscr{H}) \cap C_{00}(\mathscr{H})$ be such that $\sigma(T) \cap \mathbf{D}$ dominates $\mathbf{T}$. Then $T \in \mathbb{A}_{\aleph_{0}}^{M}(\mathscr{H})$.

\section{THE DETAILS}

In this section we prove Theorem 2 .

Since for any function $h \in \mathbf{H}^{\infty}, h(\sigma(T) \cap \mathbf{D}) \subset \sigma\left(\Phi_{T}(h)\right)$, it follows that $\Phi_{T}$ is bounded below, so $T \in \mathbb{A}^{M}(\mathscr{H})$. Thus by Theorem 3.7 of [2] it is sufficient to show that $\mathscr{A}_{T}$ has property $\mathscr{X}_{0, \theta}$ for some $\theta \in(0,1]$. The following lemma is the main ingredient in showing this.

Lemma 3. Suppose $\epsilon, \delta$ are positive numbers, $f$ is a nonnegative function in $\mathbf{L}^{1}$, and $\left\{y_{j}\right\}_{j=1}^{p}$ is a finite sequence of vectors in $\mathscr{H}$. Then there exists $x \in \mathscr{H}$ such that

$$
\left\|\phi_{T}^{-1}\left([f]_{\mathbf{L} / \mathbf{H}_{0}^{1}}\right)-[x \otimes x]_{T}\right\|<\epsilon,
$$

and

(ii) $\quad\|x\| \leq 2\|f\|_{1}^{1 / 2}, \quad\left\|\left[x \otimes y_{j}\right]_{T}\right\|+\left\|\left[y_{j} \otimes x\right]_{T}\right\|<\delta, \quad j=1, \ldots, p$.

Proof. Define $\Gamma_{1}=\left(\sigma_{p}(T) \backslash \sigma_{e}(T)\right) \cap \mathbf{D}, \Gamma_{2}=\left(\sigma(T) \backslash\left(\sigma_{p}(T) \cup \sigma_{e}(T)\right)\right) \cap \mathbf{D}$, $\widetilde{\Gamma}_{1}=N T L\left(\Gamma_{1}\right), \widetilde{\Gamma}_{2}=N T L\left(\Gamma_{2}\right)$, and $\widetilde{\Gamma}_{3}=N T L\left(\sigma_{e}(T) \cap \mathbf{D}\right)$. First we consider $f \chi_{\widetilde{\Gamma}_{1}}$. By Lemma 1.2 of [3], there exist a finite sequence of positive numbers $\left\{\alpha_{j}^{(1)}\right\}_{j=1}^{n_{1}}$ and a finite sequence $\left\{\lambda_{j}^{(1)}\right\}_{j=1}^{n_{1}}$ of distinct points in $\Gamma_{1}$, such that

$$
\sum_{j=1}^{n_{1}} \alpha_{j}^{(1)} \leq\left\|f \chi_{\widetilde{\Gamma}_{1}}\right\|_{1}
$$


and

$$
\left\|f \chi_{\widetilde{\Gamma}_{1}}-\sum_{j=1}^{n_{1}} \alpha_{j}^{(1)} P_{\lambda_{j}^{(1)}}\right\|_{1}<\epsilon / 5 .
$$

For each $j$ choose a vector of norm one $x_{j}^{(1)} \in \operatorname{ker}\left(\lambda_{j}^{(1)}-T\right)$, and define $\mathscr{H}_{1}=\operatorname{span}\left\{x_{j}^{(1)}\right\}_{j=1}^{n_{1}}$. Then $\mathscr{H}_{1} \in \operatorname{Lat}(\mathrm{T})$, and by the choice of the sequence $\left\{\lambda_{j}^{(1)}\right\}_{j=1}^{n_{1}}$, the set $\left\{x_{j}^{(1)}\right\}_{j=1}^{n_{1}}$ is linearly independent. So $\operatorname{dim} \mathscr{H}_{1}=n_{1}$, and $T_{\mathscr{K}_{1}}$ has the eigenvectors $\left\{x_{j}^{(1)}\right\}_{j=1}^{n_{1}}$ corresponding to the distinct eigenvalues $\left\{\lambda_{j}^{(1)}\right\}_{j=1}^{n_{1}}$. Therefore by Theorem 2.2 of [16] there exists $x^{(1)}$ in $\mathscr{H}_{1}$ with

$$
\left\|x^{(1)}\right\| \leq\left\|f \chi_{\widetilde{\Gamma}_{1}}\right\|_{1}^{1 / 2}
$$

such that

$$
\left[x^{(1)} \otimes x^{(1)}\right]_{T}=\sum_{j=1}^{n_{1}} \alpha_{j}^{(1)}\left[C_{\lambda_{j}^{(1)}}\right]_{T} .
$$

Hence by (3),

$$
\left\|\phi_{T}^{-1}\left(\left[f \chi_{\widetilde{\Gamma}_{2}}\right]_{\mathbf{L} / \mathbf{H}_{0}^{1}}\right)-\left[x^{(1)} \otimes x^{(1)}\right]_{T}\right\|<\epsilon / 5 .
$$

Since $\mathscr{H}_{1}$ is finite dimensional and invariant for $T$,

$$
T_{\mathscr{H} \ominus \mathscr{H}_{1}} \in(A C P B)^{M}\left(\mathscr{H} \ominus \mathscr{H}_{1}\right),
$$

$\sigma\left(T_{\mathscr{K} \ominus \mathscr{K}_{1}}\right) \cap \mathbf{D}$ dominates $\mathrm{T}, \Gamma_{1} \backslash\left\{\lambda_{j}^{(1)}\right\}_{j=1}^{n_{1}} \subset\left(\sigma_{p}\left(T_{\mathscr{H} \ominus \mathscr{H}_{1}}\right) \backslash \sigma_{e}\left(T_{\mathscr{H} \ominus \mathscr{X}_{1}}\right)\right)$, and $N T L\left(\Gamma_{1} \backslash\left\{\lambda_{j}^{(1)}\right\}_{j=1}^{n_{1}}\right)=\widetilde{\Gamma}_{1}$. By the same argument as above (applied to $T_{\mathscr{L} \ominus \mathscr{K}_{1}}$ ), one can find a finite sequence of positive numbers $\left\{\alpha_{j}^{(2)}\right\}_{j=1}^{n_{2}}$, a finite sequence $\left\{\lambda_{j}^{(2)}\right\}_{j=1}^{n_{2}}$ of distinct points in $\Gamma_{1} \backslash\left\{\lambda_{j}^{(1)}\right\}_{j=1}^{n_{1}}$, and a vector $x^{(2)}$ in $\mathscr{H} \ominus \mathscr{H}_{1}$ such that

$$
\begin{aligned}
& \left\|f \chi_{\widetilde{\Gamma}_{1}}-\sum_{j=1}^{n_{2}} \alpha_{j}^{(2)} P_{\lambda_{j}^{(2)}}\right\|_{1}<\epsilon / 5, \\
& {\left[x^{(2)} \otimes x^{(2)}\right]_{T_{x \ominus \varkappa_{1}}}=\sum_{j=1}^{n_{2}} \alpha_{j}^{(2)}\left[C_{\lambda_{j}^{(2)}}\right]_{T_{\boldsymbol{x} \ominus \boldsymbol{*}_{1}}},}
\end{aligned}
$$

and

$$
\left\|x^{(2)}\right\| \leq\left\|f \chi_{\widetilde{\Gamma}_{1}}\right\|_{1}^{1 / 2} .
$$

Since $\mathscr{K}_{1}$ is invariant for $\mathrm{T}$, by (5) it follows that

$$
\left[x^{(2)} \otimes x^{(2)}\right]_{T}=\sum_{j=1}^{n_{2}} \alpha_{j}^{(2)}\left[C_{\lambda_{j}^{(2)}}\right]_{T},
$$

and taking into account (4), we obtain

$$
\left\|\phi_{T}^{-1}\left(\left[f \chi_{\Gamma_{1}}\right]_{L / \mathbf{H}_{0}^{1}}\right)-\left[x^{(2)} \otimes x^{(2)}\right]_{T}\right\|<\epsilon / 5 .
$$

One can thus find by induction an orthogonal sequence $\left\{x^{(n)}\right\}_{n=1}^{\infty}$ such that for any positive integer $n$,

$$
\left\|x^{(n)}\right\| \leq\left\|f \chi_{\widetilde{\Gamma}_{1}}\right\|_{1}^{1 / 2}
$$


and

$$
\left\|\phi_{T}^{-1}\left(\left[f \chi_{\widetilde{\Gamma}_{1}}\right]_{\mathbf{L} / \mathbf{H}_{0}}\right)-\left[x^{(n)} \otimes x^{(n)}\right]_{T}\right\|<\epsilon / 5 .
$$

If $\mathrm{M}$ is large enough, $y^{(1)}:=x^{(M)}$ satifies the inequalities

$$
\left\|\phi_{T}^{-1}\left(\left[f \chi_{\widetilde{\Gamma}_{1}}\right]_{L / H_{0}^{1}}\right)-\left[y^{(1)} \otimes y^{(1)}\right]_{T}\right\|<\epsilon / 5,
$$

(7) $\left\|y^{(1)}\right\| \leq\left\|f \chi_{\widetilde{\Gamma}_{1}}\right\|_{1}^{1 / 2},\left\|\left[y^{(1)} \otimes y_{j}\right]_{T}\right\|+\left\|\left[y_{j} \otimes y^{(1)}\right]_{T}\right\|<\delta / 4, \quad j=1, \ldots, p$.

By a similar argument as above (applied to $f \chi_{\widetilde{\Gamma}_{2} \mid \widetilde{\Gamma}_{1}}$ ) one can obtain a vector $y^{(2)}$ such that

$$
\begin{gathered}
\left\|\phi_{T}^{-1}\left(\left[f \chi_{\widetilde{\Gamma}_{2} \mid \widetilde{\Gamma}_{1}}\right]_{\mathbf{L} / \mathbf{H}_{0}^{1}}\right)-\left[y^{(2)} \otimes y^{(2)}\right]_{T}\right\|<\epsilon / 5, \\
\left\|y^{(2)}\right\| \leq\left\|f \chi_{\widetilde{\Gamma}_{2} \mid \widetilde{\Gamma}_{1}}\right\|_{1}^{1 / 2}, \\
\left\|\left[y^{(1)} \otimes y^{(2)}\right]_{T}\right\|+\left\|\left[y^{(2)} \otimes y^{(1)}\right]_{T}\right\|<\epsilon / 5,
\end{gathered}
$$

and

$$
\left\|\left[y^{(2)} \otimes y_{j}\right]_{T}\right\|+\left\|\left[y_{j} \otimes y^{(2)}\right]_{T}\right\|<\delta / 4, \quad j=1, \ldots, p .
$$

Putting together $(6)-(11)$, we get

$$
\left\|y^{(1)}+y^{(2)}\right\| \leq 2^{1 / 2}\left\|f \chi_{\widetilde{\Gamma}_{1} \cup \widetilde{\Gamma}_{2}}\right\|_{1}^{1 / 2},
$$

$$
\left\|\left[\left(y^{(1)}+y^{(2)}\right) \otimes y_{j}\right]_{T}\right\|+\left\|\left[y_{j} \otimes\left(y^{(1)}+y^{(2)}\right)\right]_{T}\right\|<\delta / 2, \quad j=1, \ldots, p,
$$

and

$$
\left\|\phi_{T}^{-1}\left(\left[f \chi_{\widetilde{\Gamma}_{1} \cup \widetilde{\Gamma}_{2}}\right]_{\mathbf{L} / \mathbf{H}_{0}^{1}}\right)-\left[\left(y^{(1)}+y^{(2)}\right) \otimes\left(y^{(1)}+y^{(2)}\right)\right]_{T}\right\|<4 \epsilon / 5 .
$$

Now we concentrate on $f \chi_{\mathbf{T} \backslash\left(\widetilde{\Gamma}_{1} \cup \widetilde{\Gamma}_{2}\right)}$. Since $\sigma_{e}(T) \cap \mathbf{D}$ dominates $\mathbf{T} \backslash\left(\widetilde{\Gamma}_{1} \cup \widetilde{\Gamma}_{2}\right)$, again by Lemma 1.2 of [3] one can find a finite sequence of positive numbers $\left\{\alpha_{k}\right\}_{k=1}^{L}$, and a finite sequence $\left\{\lambda_{k}\right\}_{k=1}^{L} \subset \sigma_{e}(T)$ such that

$$
\sum_{k=1}^{L} \alpha_{k} \leq\left\|f \chi_{\mathbf{T} \backslash\left(\tilde{\Gamma}_{1} \cup \widetilde{\Gamma}_{2}\right)}\right\|_{1},
$$

and

$$
\left\|\phi_{T}^{-1}\left(\left[f \chi_{\mathbf{T} \backslash\left(\widetilde{\Gamma}_{1} \cup \widetilde{\Gamma}_{2}\right)}\right]_{\mathbf{L} / \mathbf{H}_{0}^{1}}\right)-\sum_{k=1}^{L} \alpha_{k}\left[C_{\lambda_{k}}\right]_{T}\right\|<\epsilon / 20 .
$$

For each $k \in\{1, \ldots, L\}$ let $\left\{x_{n}^{(k)}\right\}_{n=1}^{\infty}$ be a sequence of vectors in the unit ball of $\mathscr{H}$, converging weakly to 0 such that

$$
\lim _{n \rightarrow \infty}\left\|\left[C_{\lambda_{k}}\right]_{T}-\left[x_{n}^{(k)} \otimes x_{n}^{(k)}\right]_{T}\right\|=0 .
$$

By a standard argument (since $\lim _{n \rightarrow \infty}\left(\left\|\left[x_{n}^{(k)} \otimes u\right]_{T}\right\|+\left\|\left[u \otimes x_{n}^{(k)}\right]_{T}\right\|\right)=0$ for any $u$ in $\mathscr{H}, k=1, \ldots, L)$ one can choose inductively positive integers $\left\{n_{k}\right\}_{k=1}^{L}$ such that

$$
y^{(3)}:=\sum_{k=1}^{L} \alpha_{k}^{1 / 2} x_{n_{k}}^{(k)}
$$


satisfies

$$
\begin{gathered}
\left\|\phi_{T}^{-1}\left(\left[f \chi_{\mathbf{T} \backslash\left(\widetilde{\Gamma}_{1} \cup \widetilde{\Gamma}_{2}\right)}\right]_{\mathbf{L} / \mathbf{H}_{0}^{1}}\right)-\left[y^{(3)} \otimes y^{(3)}\right]_{T}\right\|<\epsilon / 10, \\
\left\|y^{(3)}\right\| \leq 2^{1 / 2}\left\|f \chi_{\mathbf{T} \backslash\left(\widetilde{\Gamma}_{1} \cup \widetilde{\Gamma}_{2}\right)}\right\|_{1}^{1 / 2}, \\
\left\|\left[\left(y^{(1)}+y^{(2)}\right) \otimes y^{(3)}\right]_{T}\right\|+\left\|\left[y^{(3)} \otimes\left(y^{(1)}+y^{(2)}\right)\right]_{T}\right\|<\epsilon / 20,
\end{gathered}
$$

and

$$
\left\|\left[y^{(3)} \otimes y_{j}\right]_{T}\right\|+\left\|\left[y_{j} \otimes y^{(3)}\right]_{T}\right\|<\delta / 2, \quad j=1, \ldots, L .
$$

By (12)-(18) it follows easily that the vector $x:=y^{(1)}+y^{(2)}+y^{(3)}$ satisfies (i) and (ii) above, and the lemma is proved.

The next step in the proof of Theorem 2 is to show that if $f$ is a nonnegative function in $\mathbf{L}^{1}$ such that $\|f\|_{1} \leq 1 / 2$, then $\phi_{T}^{-1}\left([f]_{\mathbf{L} / \mathbf{H}_{0}^{1}}\right) \in \mathscr{E}_{0}\left(\mathscr{A}_{T}\right)$. Once this has been shown, it will follow that if $f \in \mathbf{L}^{1}$ is such that $\|f\|_{1} \leq 1 / 8$, then $\phi_{T}^{-1}\left([f]_{\mathbf{L} / \mathbf{H}_{0}^{1}}\right) \in \mathscr{E}_{0}\left(\mathscr{A}_{T}\right)$. Thus taking into account the facts that $\phi_{T}$ is invertible and $\left\|[f]_{\mathbf{L} / \mathbf{H}_{0}^{1}}\right\| \leq M\left\|\phi_{T}^{-1}\left([f]_{\mathbf{L} / \mathbf{H}_{0}^{1}}\right)\right\|$ for any $f \in \mathbf{L}^{1}$, it will follow that $\mathscr{A}_{T}$ has property $\mathscr{X}_{0,1 / 8 M}$, so we are done. To see that for any $f \in \mathbf{L}^{1}$ such that $\|f\|_{1} \leq 1 / 8, \phi_{T}^{-1}\left([f]_{\mathbf{L} / \mathbf{H}_{0}^{1}}\right) \in \mathscr{E}_{0}\left(\mathscr{A}_{T}\right)$, pick two sequences of positive numbers $\left\{\epsilon_{n}\right\}_{n=1}^{\infty}$ and $\left\{\delta_{n}\right\}_{n=1}^{\infty}$ decreasing to 0 , and a dense, countable subset $\left\{z_{n}\right\}_{n=1}^{\infty}$ in $\mathscr{H}$. By Lemma 2 , one can find a sequence $\left\{x^{(n)}\right\}_{n=1}^{\infty}$ of vectors in the unit ball of $\mathscr{H}$ such that for every $n$,

$$
\left\|\phi_{T}^{-1}\left([f]_{\mathbf{L} / \mathbf{H}_{0}^{1}}\right)-\left[x^{(n)} \otimes x^{(n)}\right]_{T}\right\|<\epsilon_{n}
$$

and

$$
\left\|\left[x^{(n)} \otimes z_{k}\right]_{T}\right\|+\left\|\left[z_{k} \otimes x^{(n)}\right]_{T}\right\|<\delta_{n}, \quad k=1, \ldots, n,
$$

so the sequence $\left\{x^{(n)}\right\}_{n=1}^{\infty}$ converges weakly to 0 . Hence by Lemma 1 , $\phi_{T}^{-1}\left([f]_{\mathbf{L} / \mathbf{H}_{0}^{1}}\right) \in \mathscr{E}_{0}\left(\mathscr{A}_{T}\right)$, and the proof of the theorem is complete.

Remarks. This paper constitutes part of the author's Ph.D. thesis written at Texas A\&M University under the direction of Carl Pearcy. The referee has kindly pointed out that Jörg Eschmeier obtained a similar result in [10].

\section{REFERENCES}

1. H. Bercovici, A contribution to the theory of operators in the class $\mathbb{A}$, J. Funct. Anal. 78 (1988), 197-207.

2. H. Bercovici, C. Foias, and C. Pearcy, Dual algebras with applications to invariant subspaces and dilation theory, CBMS Regional Conf. Ser. in Math., vol. 56, Amer. Math. Soc., Providence, RI, 1985.

3. H. Bercovici, C. Foiaş, C. Pearcy, and B. Sz.-Nagy, Functional models and extended spectral dominance, Acta Sci. Math. (Szeged) 43 (1981), 243-254.

4. H. Bercovici, C. Foiaş, and C. Pearcy, Two Banach space methods and dual operator algebras, J. Funct. Anal. 78 (1988), 306-346.

5. S. Brown and B. Chevreau, Toute contraction à calcul fonctionnel isométrique est réflexive, C. R. Acad. Sci. Paris Sér. I Math. 307 (1988), 185-188.

6. S. Brown, B. Chevreau, and C. Pearcy, On the structure of contraction operators. II, J. Funct. Anal. 76 (1988), 30-55. 
7. B. Chevreau, G. Exner, and C. Pearcy, On the structure of contraction operators. III, Michigan Math. J. 36 (1989), 29-62.

8. B. Chevreau, C. Pearcy, and A. Shields, Finitely connected domains $G$, representations of $H^{\infty}(G)$, and invariant subspaces, J. Operator Theory 6 (1981), 123-136.

9. J. Dixmier, Les algèbres d'opérateurs dans l'espace Hilbertien (Algèbres de von Neumann), Gauthier-Villars, Paris, 1957.

10. J. Eschmeier, Representations of $\mathbf{H}^{\infty}(G)$ and invariant subspaces, preprint.

11. W. S. Li, On polynomially bounded operators, Ph.D. Thesis, Michigan.

12. __ On polynomially bounded operators. I, Houston J. Math. 18 (1992), 73-96.

13. $ـ$ On polynomially bounded operators. II, preprint.

14. B. Sz.-Nagy and C. Foiaş, Harmonic analysis of operators on Hilbert space, North-Holland, Amsterdam, 1970.

15. V. Paulsen, C. Pearcy, and S. Petrovic, On centered and weakly centered operators, J. Funct. Anal. (to appear).

16. D. Westood, On $C_{00}$-contractions with dominating spectrum, J. Funct. Anal. 66 (1986), 96-104.

Department of Mathematics, Texas A\&M University, College Station, Texas 77843

Current address: Department of Mathematics, Case Western Reserve University, Cleveland, Ohio 44106/7058

E-mail address: rxg38@po.cwru.edu 